\title{
Fundamental physical processes in coronae: Waves, turbulence, reconnection, and particle acceleration
}

\author{
Markus J. Aschwanden ${ }^{1}$ \\ ${ }^{1}$ Laboratory for Solar and Astrophysics, ATC, Lockheed Martin, Bldg. 252, Org. ADBS, Palo \\ Alto, CA 94304, USA \\ email: aschwanden@lmsal.com
}

\begin{abstract}
Our understanding of fundamental processes in the solar corona has been greatly progressed based on the space observations of SMM, Yohkoh, Compton GRO, SOHO, TRACE, RHESSI, and STEREO. We observe now acoustic waves, MHD oscillations, turbulence-related line broadening, magnetic configurations related to reconnection processes, and radiation from high-energy particles on a routine basis. We review a number of key observations in EUV, soft $\mathrm{X}$-rays, and hard X-rays that innovated our physical understanding of the solar corona, in terms of hydrodynamics, MHD, plasma heating, and particle acceleration processes.
\end{abstract}

Keywords. Waves, Turbulence, Reconnection, Particle Acceleration

\section{Introduction}

Our grasp to understand the fundamental physical processes in the solar corona in more depth mostly benefits from the new high-resolution imaging capabilities that became available in extreme ultraviolet (EUV), soft X-rays, hard X-rays, and gamma rays. These wavelengths are particularly revealing in the following aspects: The corona is filled with high-temperature plasma at temperatures of $T \approx 1-2 \mathrm{MK}$, which radiates freefree emission with its emission measure peak in EUV wavelengths. Hot active regions and postflare loops have plasma temperatures of $T \approx 2-40 \mathrm{MK}$ with their free-free emission peak in soft X-ray wavelengths. The nonthermal emission of flare-accelerated high-energy particles radiate bremsstrahlung in hard X-ray wavelengths and have nuclear de-excitation lines in gamma rays. Because of the absorbing properties of the Earth atmosphere, the Sun can be observed in these wavelengths only from space.

We discovered waves in the solar corona from oscillatory plasma motions and from propagating density disturbances. Turbulence is somewhat harder to detect, but we mostly infer if from line broadening in EUV or from scintillation experiments in radio wavelengths. Magnetic reconnection is most conspicuously inferred from the postreconnection topology of post-flare loop systems, but we detect also plasma inflows and outflows in reconnection regions. Particle acceleration is still the biggest black box because it occurs on microscopic scales, but we can localize the acceleration regions from direct bremsstrahlung in the acceleration region itself, or from reconstructed particle trajectories using time-of-flight measurements. Here we review the fundamental physics of these four processes, applied to solar and stellar coronae, and touch on some new challenging observations. 


\section{Waves in Coronae}

Waves and acoustics have been established as fundamental fields in physics since the days of Pythagoras, Isaac Newton, and Christian Huygens. Wave phenomena are governed by an oscillatory dynamics between two competing forces, such as the inertial motion and the restoring force of a mechanical elastic medium, or the electric and magnetic field in an electromagnetic wave. In the solar corona we have magnetic field lines that are filled with plasma like fluxtubes, which are governed by magnetohydrodynamics (MHD) and can exhibit propagating and standing waves. These MHD wave modes can be derived from the so-called ideal MHD equations, by inserting the current density $\mathbf{j}=(1 / 4 \pi) \nabla \times \mathbf{B}$, the electric field $\mathbf{E}=(-1 / c) \mathbf{v} \times \mathbf{B}$ from Ohm's law, the definition of the sound speed $c_{S}=\gamma p / \rho$, and assuming an adiabatic process with polytropic index $\gamma$, $\nabla p=c_{S}^{2} \nabla \rho$, which leads to the dispersion relation of MHD waves, expressed in terms of the three variables $(\rho, \mathbf{v}, \mathbf{B})$, i.e., the mass density $\rho$, the velocity $\mathbf{v}$, and the magnetic field strength $\mathbf{B}$. Linearizaton of the equations for small perturbations in $(\rho, \mathbf{v}, \mathbf{B})$ around a stationary solution, neglecting the gravity $g$, using the definition of the Alfvén speed $v_{A}=B_{0} / \sqrt{4 \pi \rho_{0}}$, and inserting the Fourier form $(d / d t \mapsto i \omega, \nabla \mapsto i k)$ yields then the well-known dispersion relation for magneto-acoustic waves,

$$
\omega^{4}-k^{2}\left(c_{S}^{2}+v_{A}^{2}\right) \omega^{2}+k_{z}^{2} k^{2} c_{S}^{2} v_{A}^{2}=0,
$$

which has two branches of solutions, called fast and slow magneto-acoustic waves. For the non-magnetic case $\left(B=0\right.$ and $\left.v_{A}=0\right)$, the phase speed is equal to the sound speed, $v_{p h}=c_{S}$, which is the slow or acoustic mode, producing a longitudinal wave, is non-dispersive, and compressional $\left(\rho_{1} \propto v_{1}\right)$. In a magnetized plasma $(B \neq 0)$, the phase speed depends on the angle between the magnetic field $\mathbf{B}$ and the wave propagation vector $\mathbf{k}$ and is called the fast mode. For the special case of a perpendicular angle $\theta=90^{\circ}$, called a shear Alfvén wave, the phase speed is $v_{p h}=\sqrt{c_{S}^{2}+v_{A}^{2}}$, and the perpendicular wave is dispersive and incompressible $\left(B_{1} \propto v_{1}\right)$.

These basic properties tell us already how these waves are detected in the solar corona. Acoustic waves show up as pressure fluctuations and thus can be detected from density fluctuations. Optically thin emission in EUV and soft X-rays has an emission measure that is proportional to the square of the electron density, $E M \propto \int n_{e}^{2}(z) d z$, and thus propagating sound waves can be traced from the associated emission measure variations. A further proof of the identity of detected acoustic waves is the measurement of the propagation speed, which turned out to be identical to the sound speed $c_{S} \approx 150 T_{M K}$ $\mathrm{km} \mathrm{s}^{-1}$ expected for the observed temperature of the plasma (i.e., $T \approx 1.0 \mathrm{MK}$ at $171 \AA$ ). Such slow (acoustic) waves have been detected by DeForest \& Gurman (1998), Berghmans \& Clette (1999), DeMoortel et al. (2000; 2002), and Robbrecht et al. (2001), using SOHO/EIT and TRACE (Table 1), and have even been re-discovered in Yohkoh data (Mariska 2005, 2006). These propagating acoustic waves were all detected in upward direction in open magnetic field structures, where no reflection occurs. In closed-field structures, acoustic waves seem to be excited at one footpoint by some flare-like disturbance, which then propagate with sound speed to the opposite footpoint and are reflected, building up a standing (acoustic) oscillation or eigen-mode. Such acoustic oscillations, detected from the Dopplershift of the velocity disturbance as well as from the density variations, have been reported from SOHO/SUMER observations (Wang et al. 2002; Kliem et al. 2002), with periods of order $P \approx 10-20$ min (Table 1 ).

The detection and identification of fast MHD waves is quite different from acoustic modes because of their different physical properties. Let us consider the fast MHD standing waves (or eigen-modes). The fast kink mode is an asymmetric mode and shows 


\begin{tabular}{|c|c|c|c|}
\hline MHD wave type & Period range & Observations & References of examples \\
\hline \multicolumn{4}{|l|}{ MHD Oscillations } \\
\hline$\overline{\text { Fast kink mode }}$ & $\approx 3-5 \min$ & TRACE & Aschwanden et al. (1999) \\
\hline \multirow{3}{*}{ Fast sausage mode } & $\approx 1-10 \mathrm{~s}$ & Radio & $\begin{array}{l}\text { Nakariakov et al. (1999) } \\
\text { Aschwanden (1987) }\end{array}$ \\
\hline & & Nobeyama & Asai et al. (2001) \\
\hline & & Nobeyama & Melnikov et al. $(2002,2005)$ \\
\hline \multirow[t]{2}{*}{ Slow (acoustic) mode } & $\approx 10-20 \mathrm{~min}$ & SOHO/SUMER & Wang et al. (2002) \\
\hline & & SOHO/SUMER & Kliem et al. (2002) \\
\hline Propagating MHD Waves & Velocity range & & \\
\hline \multirow[t]{4}{*}{$\overline{\text { Slow (acoustic) waves }}$} & $75-150 \mathrm{~km} / \mathrm{s}$ & $\mathrm{SOHO} / \mathrm{EIT}$ & DeForest \& Gurman (1998) \\
\hline & $75-200 \mathrm{~km} / \mathrm{s}$ & SOHO/EIT & Berghmans \& Clette (1999) \\
\hline & $70-235 \mathrm{~km} / \mathrm{s}$ & TRACE & DeMoortel et al. $(2000,2002)$ \\
\hline & $65-150 \mathrm{~km} / \mathrm{s}$ & TRACE, SOHO/EIT & Robbrecht et al. (2001) \\
\hline \multirow[t]{2}{*}{ Fast (Alfvénic) waves } & $2100 \mathrm{~km} / \mathrm{s}$ & SECIS & $\begin{array}{l}\text { Williams et al. }(2001) \\
\text { Katsiyannis et al. }(2003)\end{array}$ \\
\hline & $1000-4000 \mathrm{~km} / \mathrm{s}$ & $\mathrm{CMCP} / \mathrm{NSO}$ & Tomczyk et al. 2007) \\
\hline Fast kink waves & $100-500 \mathrm{~km} / \mathrm{s}$ & TRACE & Verwichte et al. (2005) \\
\hline
\end{tabular}

Table 1. MHD wave types identified in the solar corona.

perpendicular displacements of the loop centroid, but only with relatively small amplitudes in the order of a few percent of the loop length. Only in 1999, after the highresolution EUV images of TRACE with a spatial resolution of $\approx 1^{\prime \prime}$ became available, such fast kink modes were discovered (Aschwanden et al. 1999; Nakariakov et al. 1999). Theoretically, these fast kink modes could have been detected with SOHO/EIT earlier, but apparently the image cadence was never chosen to be sufficiently fast to resolve the typical kink mode periods of $P \approx 3-5 \mathrm{~min}$.

The other branch of fast MHD modes is the sausage mode, a symmetric mode that should show up as a radial oscillation, and thus the emission measure should be modulated by the 4 th power of the wave amplitude, i.e., $\Delta E M(t) / E M \propto 4 \Delta r(t) / r$. Although there was a lot of evidence for fast MHD oscillations in terms of the expected fast periods in the order of $P \approx 1-10 \mathrm{~s}$ from non-imaging radio observations (e.g., see review of Aschwanden 1987), there is still rather sparse evidence from imaging observations. One case attributed to the fast sausage mode has been reported from Nobeyama observations (Asai et al. 2001; Melnikov et al. 2002, 2005; Nakariakov et al. 2003), based on periods from hard X-ray and microwave light curves and the estimated Alfvén crossing time. However, the emission measure modulation and spatially-resolved radial oscillations of the thermal plasma associated with the fast sausage MHD mode have never been directly observed. The theory predicts an anti-correlated variation of the cross-sectional loop radius with its electron density, which should be detectable with current soft X-ray imagers (such as HINODE/XRT). Moreover, the fast sausage mode is also subject to a wavenumber cutoff at a phase speed of $v_{p h}=v_{A e}$ (when the phase speed matches the external Alfvén speed), which implies relatively fat loops (which a large width/length ratio $w / l$ ) or very dense loops. This requirement for the density contrast between the internal and the external density, $n_{0} / n_{e}$,

$$
\frac{n_{0}}{n_{e}} \gtrsim 2.334\left(\frac{l}{w}\right)^{2},
$$

is especially restrictive for slender loops (say $l / w>10$ ), which requires very overdense loops $\left(n_{0} / n_{e}>230\right)$ that only exist in post-flare conditions (Aschwanden et al. 2004). 
The detection of such fast sausage modes with periods of a few seconds from the thermal plasma is still difficult because sub-second cadences of soft X-ray imagers are rarely available.

The detection of propagating fast Alfvénic waves is also difficult, because a purely Alfvénic wave is incompressible and does not produce any modulation of the density that could be easily observed. Alfvénic waves cause a modulation $B_{1} \propto v_{1}$, but the magnetic field fluctuations $B_{1}$ cannot be measured by current methods, while fluctuations of velocities $v_{1}$ can only be measured from line broadening, but are subject to interpretational ambiguities (such as in terms of turbulence). However, there is a continuous range of intermediate magneto-acoustic waves between the slow (acoustic) mode and the fast (Alfvénic) mode, which display a mixed characteristic of compressible and incompressible waves, which leaves a possibility for detection by density modulations. Claims for detected fast Alfvénic waves in the solar corona come mainly from the phase speed of observed disturbances, which were found to be of order $v_{p h} \approx 2100 \mathrm{~km} \mathrm{~s}^{-1}$ from high-cadence optical images during a solar eclipse (Williams et al. 2001; Katsiyannis et al. 2003), and of order $v_{p h} \approx 1000-4000 \mathrm{~km} \mathrm{~s}^{-1}$ from a Coronal Multi-Channel Polarimeter at NSO (Tomczyk et al. 2007).

There are also exciting observations of oscillations from flare stars. Gary et al. (1982) reported oscillations with periods of $P=56 \mathrm{~s}$ at $9.4 \mathrm{GHz}$ from the flare star UV Ceti, which probably is coherent radio emission modulated by the magnetic field variations of a fast MHD mode. There are reports of oscillations with periods of $P=9-246 \mathrm{~s}$ in optical wavelengths (Andrews 1990; Mullan et al. 1992; Mathioudakis et al. 2003) which are harder to explain because we do not understand how MHD modes modulate optical emission. And there is a number of observed stellar oscillations in soft X-rays with periods of $P=56-750 \mathrm{~s}$ (Mullan \& Johnson 1995; Mitra-Kraev et al. 2005), which have all faster periods than what we are used to see for slow acoustic modes in the Sun, with typical periods of $P \approx 10-20 \mathrm{~min}$, at a temperature of $T=7 \mathrm{MK}$ measured with SUMER/SOHO. However, since stellar flares have significantly higher temperatures, in the range of $T=10-100 \mathrm{MK}$, the acoustic speed is also faster, in the order of $c_{S}=450-1500 \mathrm{~km} \mathrm{~s}^{-1}$, which could explain faster periods of standing acoustic waves.

Thus, our observations of MHD standing and propagating waves in the solar corona become more complete, but some of the waves modes are still difficult to detect due to insufficient cadence, spatial resolution, and sensitivity. In stellar flares we lack the spatial resolution, and thus cannot determine the loop length that is important to infer the acoustic or Alfvénic loop crossing time, and thus have to rely on applications of scaling laws from solar flares. For a review of other recent work on the topic of waves in coronae (omitted here) see also review by Nakariakov in this book.

\section{Turbulence in Coronae}

Convection and turbulence are important in fluids with high Reynolds numbers. Since the magnetic Reynolds number $R_{m}=l_{0} v_{0} / \eta_{m}$ (or Lundquist number) is high in the coronal plasma $\left(R_{m} \approx 10^{8}-10^{12}\right)$, turbulence may also develop in coronal loops (although there is the question whether turbulence could be suppressed in coronal loops due to the photospheric line-tying). Theoretical models and numerical simulations that study MHD turbulence include the kinematic viscosity or shear viscosity $\nu_{v i s c}$ in the MHD momentum equation,

$$
\rho \frac{D \mathbf{v}}{D t}=-\nabla p-\rho \mathbf{g}+(\mathbf{j} \times \mathbf{B})+\nu_{v i s c} \rho\left[\nabla^{2} \mathbf{v}+\frac{1}{3} \nabla(\nabla \cdot \mathbf{v})\right]
$$


and the magnetic diffusivity $\eta_{m}=c^{2} / 4 \pi \sigma$ in the MHD induction equation,

$$
\frac{\partial \mathbf{B}}{\partial t}=\nabla \times(\mathbf{v} \times \mathbf{B})+\eta_{m} \nabla^{2} \mathbf{B}
$$

Similar to the models of stress-induced current cascades, random footpoint motion is assumed to pump energy into a system at large scales (into eddies the size of a granulation cell, $\approx 1000 \mathrm{~km}$ ), which cascade due to turbulent motion into smaller and smaller scales, where the energy can be more efficiently dissipated by friction, which is quantified by the kinematic or shear viscosity coefficient $\nu_{v i s c}$. Friction and shear are dynamical effects resulting from the nonlinear terms $\left(v_{1, i} v_{1, j}\right),\left(v_{1, i} B_{1, j}\right),\left(B_{1, i} v_{1, j}\right)$, and $\left(B_{1, i} B_{1, j}\right)$ in Eqs. (3.1-3.2) and are only weakly sensitive to the detailed dynamics of the boundary conditions. Analytical (3D) models of MHD turbulence have been developed by Heyvaerts \& Priest (1992), Inverarity et al. (1995), Inverarity \& Priest (1995), and Milano et al. (1997), where the nonlinear viscosity terms are specified as diffusion coefficients. These turbulent diffusion coefficients are free parameters, which are constrained selfconsistently by (1) assuming that the random footpoint motion has a turbulence power spectrum (e.g., a Kolmogorov spectrum $P(k) \propto k^{5 / 3}$ ); and (2) by matching the observed macroscopic parameters (i.e., velocity of footpoint motion, density, and magnetic field). Heyvearts \& Priest (1992) predict turbulent velocities of $v_{\text {turb }} \approx 20-30 \mathrm{~km} \mathrm{~s}^{-1}$, which are consistent with the excess broadening of lines observed with SUMER, which shows a peak of $\xi=30 \mathrm{~km} \mathrm{~s}^{-1}$ at a transition region temperature of $T \approx 3 \times 10^{5} \mathrm{~K}$ (e.g., Chae et al. 1988).

Analytical models of turbulent heating are applied to sheared arcades (Inverarity et al. 1995) and twisted fluxtubes (Inverarity \& Priest 1995). Turbulent heating has been numerically simulated in a number of studies, which exhibit a high degree of spatial and temporal intermittency (Einaudi et al. 1996a, b; Dmitruk \& Gomez 1997). Such simulations show how larger eddies fragment into smaller ones, forming current sheets and triggering magnetic reconnection during this process. Heating occurs by Ohmic dissipation in the thinnest current sheets. Milano et al. (1999) emphasize that the locations of heating events coincide with quasi-separatrix layers. The formation of such current sheets has also been analytically studied in the context of turbulent heating by Aly \& Amari (1997). Numerical simulations reveal intermittent heating events with energies of $E_{H}=5 \times 10^{24}$ to $10^{26}$ erg and a frequency distribution with a powerlaw slope of $\alpha \approx 1.5$, similar to observed nanoflare distributions in EUV (Dmitruk \& Gomez 1997; Dmitruk al. 1998).

Although we have a lot of theoretical modeling and MHD simulations on the fundamental process of turbulence, the major question we face is what is the observational evidence that this process operates in the solar corona, and how can it be tested observationally. One of the key observables that is thought to provide evidence for turbulence is the nonthermal line broadening in EUV and soft X-ray lines. However, the detection of nonthermal line broadening is not a sufficient criterion, because it can also be caused by unresolved flows that are not necessarily due to turbulence. An additional constraint could be the altitude dependence of the nonthermal excess line broadening $\xi(T)$, which was found to increase from $\xi \approx 5 \mathrm{~km} \mathrm{~s}^{-1}$ in the temperature minimum region $\left(T \approx 10^{4} \mathrm{~K}\right)$ to a maximum of $\xi \approx 30 \mathrm{~km} \mathrm{~s}^{-1}$ in the transition region $\left(T \approx 3 \times 10^{5} \mathrm{~K}\right)$, and to decrease further upwards in the lower corona (e.g., Chae et al. (1998). Equating the heating rate to the turbulent dissipation rate, Chae et al. (1998) was able to reproduce the observed height and temperature dependence of the nonthermal line broadening. Implicitly, this model implies, however, that the heating rate is concentrated in the transition region 
and becomes negligible in the coronal part of loops. The hypothesis of footpoint heating, moreover, is also supported by at least ten other observational constraints (Aschwanden 2001, Aschwanden et al. 2007).

On the other side, the identification of turbulence has been clearly established in the outer or extended corona. The solar wind is considered as a medium with welldeveloped MHD turbulence, as shown by the coupled variations of the three magnetic field components and the fluid velocity (Belcher \& Davis 1971), which is expected for Alfvén waves $\left(v_{1} \propto B_{1}\right)$. In the framework of the "MHD turbulent cascade", energy is injected at large scales and converted into smaller scales (or eddies) with subsequent dissipation at the smallest scales, resulting into plasma heating (Goldstein et al. 1995). The inertial range of the turbulent power spectrum has a powerlaw slope of $3 / 2$ or $5 / 3$ (Kraichnan or Kolmogorov spectrum), as it is observed in the interplanetary magnetic field (Leamon et al. 1998). The current thinking is that the energy input into the fast solar wind comes from kink-mode field motion, generated by transverse shaking in intergranular lanes, which is then transformed into Alfvén waves in the canopies of the transition region and propagates into the fast solar wind. A new insight is that the upward propagating Alfvén waves become partially reflected in the solar wind, so that MHD turbulence develops from the nonlinear interaction of outward and inward propagating Alfvén waves (Cranmer \& van Ballegooijen 2005). The heating of the solar wind is then accomplished by ioncyclotron wave-particle interactions of these Alfvén waves, as it has been convincingly demonstrated by the surprisingly large ion temperatures, ion outflow speeds $\left(\mathrm{H}^{\circ}, \mathrm{O}^{5+}\right)$, and velocity distribution anisotropies of positive ions observed by SOHO/UVCS above coronal holes in the solar wind at distances of $\approx 1-3$ solar radii ( $\mathrm{Li}$ et al. 1998).

In summary, in the solar context, turbulence seems to play an important role in the transition region and in the extended corona (solar wind), while there is less evidence for heating by turbulence in the closed-field corona.

\section{Magnetic Reconnection in Coronae}

Theory and numerical simulations of magnetic reconnection processes in the solar corona have been developed for steady $2 \mathrm{D}$ reconnection, bursty $2 \mathrm{D}$ reconnection, and $3 \mathrm{D}$ reconnection. Only steady $2 \mathrm{D}$ reconnection models can be formulated analytically, which provide basic relations for inflow speed, outflow speed, and reconnection rate, but represent oversimplifications for most (if not all) observed flares. A more realistic approach seems to be bursty $2 \mathrm{D}$ reconnection models, which involve the tearing-mode and coalescence instability and can reproduce the sufficiently fast temporal and small spatial scales required by solar flare observations. The sheared magnetic field configurations and the existence or coronal and chromospheric nullpoints, which are now inferred more commonly in solar flares, require ultimately 3D reconnection models, possibly involving nullpoint coalescence, spine reconnection, fan reconnection, and separator reconnection. Magnetic reconnection operates in two quite distinct physical parameter domains (in collisional or collisionless plasma): (i) in the chromosphere during magnetic flux emergence, magnetic flux cancellation, and so-called explosive events, and (ii) under coronal conditions during microflares, flares, and CMEs. The best known flare/CME models entail magnetic reconnection processes that are driven by a rising filament/prominence, by flux emergence, by converging flows, or by shear motion along the neutral line. Flare scenarios with a driver perpendicular to the neutral line (rising prominence, flux emergence, convergence flows) are formulated as 2D reconnection models (Kopp \& Pneuman 1976; Heyvaerts et al. 1977; Forbes \& Priest 1995; Uchida 1980), while scenarios that involve shear along the neutral line (tearing-mode instability, quadrupolar flux transfer, the 


\begin{tabular}{|c|c|c|}
\hline Physical aspect: & Observational signature: & References: \\
\hline $\mathrm{X}$-point geometry & Cusp in LDE events & Tsuneta et al. (1992) \\
\hline X-point altitude & $\begin{array}{l}\text { Time-of-flight measurements } \\
\text { Above-the-looptop HXR sources }\end{array}$ & $\begin{array}{l}\text { Aschwanden et al. (1996) } \\
\text { Masuda et al. (1994) }\end{array}$ \\
\hline $\mathrm{X}$-point rises with time & $\begin{array}{l}\text { Increasing footpoint separation } \\
\text { or double-ribbon separation }\end{array}$ & $\begin{array}{l}\text { Sakao et al. (1998) } \\
\text { Fletcher \& Hudson (2001) }\end{array}$ \\
\hline X-point symmetry, horizontal & $\begin{array}{l}\text { Simultaneous HXR emission } \\
\text { at conjugate footpoints }\end{array}$ & Sakao et al. (1994) \\
\hline $\mathrm{X}$-point symmetry, vertical & $\begin{array}{l}\text { Bi-directional type III bursts } \\
\text { Coincidence HXR + type III } \\
\text { Dual coronal HXR sources }\end{array}$ & $\begin{array}{l}\text { Aschwanden et al. (1995) } \\
\text { Aschwanden et al. (1993) } \\
\text { Sui \& Holman (2003) }\end{array}$ \\
\hline Post-reconnection relaxation & $\begin{array}{l}\text { Loop shrinkage ratio } \\
\text { cooling loops below hot loops }\end{array}$ & $\begin{array}{l}\text { Forbes \& Acton (1996) } \\
\text { Svestka et al. }(1987)\end{array}$ \\
\hline Quadrupolar geometry & Interacting flare loops & $\begin{array}{l}\text { Hanaoka }(1996,1997) \\
\text { Nishio et al. }(1997) \\
\text { Aschwanden et al. }(1999 \mathrm{a})\end{array}$ \\
\hline 3D nullpoint geometry & Fan dome and spine morphology & Fletcher et al. (2001) \\
\hline Reconnection inflows & EUV inward motion & Yokoyama et al. (2001) \\
\hline Reconnection outflows & Supra-arcade downflows & McKenzie \& Hudson (1999) \\
\hline Slow-mode standing shocks & High-temperature ridges & Tsuneta (1996) \\
\hline Fast-mode standing shocks & $\begin{array}{l}\text { High density above looptop } \\
\text { Above-the-looptop HXR }\end{array}$ & $\begin{array}{l}\text { Tsuneta et al. (1997) } \\
\text { Masuda et al. (1994) }\end{array}$ \\
\hline Plasmoid ejection & $\begin{array}{l}\text { Upward-moving plasmoid } \\
\text { Streamer blobs }\end{array}$ & $\begin{array}{l}\text { Shibata et al. }(1992) \\
\text { Sheeley et al. }(1997)\end{array}$ \\
\hline Downward conduction & Downward thermal fronts & Rust et al. (1985) \\
\hline Chromospheric evaporation & $\begin{array}{l}\text { Line broadening } \\
\text { SXR upflows } \\
\text { SXR blueshifts } \\
\text { H } \alpha \text { redshifts } \\
\text { Momentum balance } \\
\text { HXR } / \mathrm{H} \alpha \text { ribbons }\end{array}$ & $\begin{array}{l}\text { Antonucci et al. }(1986) \\
\text { Acton et al. }(1982) \\
\text { Czaykowska et al. }(1999) \\
\text { Zarro \& Canfield (1989) } \\
\text { Wuelser et al. }(1994) \\
\text { Hoyng et al. }(1981)\end{array}$ \\
\hline
\end{tabular}

Table 2. Key observations that provide evidence for magnetic reconnection in solar flares and CMEs (Aschwanden 2004).

magnetic breakout model, sheared arcade interactions) require 3D descriptions (Sturrock 1966; Antiochos et al. 1999; Somov et al. 1998). Ultimately, most of these partial flare models could be unified in a 3D model that includes all driver mechanisms. Observational evidence for magnetic reconnection in flares includes the 3D geometry, reconnection inflows, outflows, detection of shocks, jets, ejected plasmoids, and secondary effects like particle acceleration, conduction fronts, and chromospheric evaporation processes (summarized in Table 2). Magnetic reconnection not only operates locally in flares, it also organizes the global corona by large-scale restructuring processes.

Let us mention a few recent studies that stimulate and challenge our thinking about magnetic reconnection processes in solar flares. Dual coronal hard X-ray sources with vertically symmetric energy sources have been discovered with RHESSI, which for the 
first time directly demonstrate the vertical symmetry of an X-type reconnection geometry (Sui \& Holman 2003). A challenging phenomenon that has been observed in a few flares is the initial altitude drop of the coronal hard X-ray source before it rises in the later flare phase (Sui \& Holman 2003; Asai et al. 2004). Is it related to the relaxation of newlyreconnected field lines or to the local implosion after a CME launch? The spatial evolution of reconnection in a flare arcade was found to be quite complex: sometimes it can be tracked from footpoint motions that increase in separation and move systematically along the ribbons (Krucker et al. 2003), and sometimes they move along ribbons rather than apart as predicted by the Kopp-Pneuman model (Grigis \& Benz 2005). Important insight into the reconnection process was also found from determining the reconnection rate via the footpoint velocity and the local footpoint magnetic field (Asai et al. 2004a). The energy release rate can then be obtained from the product of the Poynting flux and the area of the reconnection region $(A)$,

$$
\frac{d E}{d t}=2 \frac{B_{c}^{2}}{4 \pi} v_{i} A
$$

which was found to correlate with the hard X-ray flux (Asai et al. 2004a; Krucker et al. 2005). RHESSI continues to provide important physical parameters to disentangle the spatial and temporal evolution of magnetic reconnection processes.

\section{Particle Acceleration in Coronae}

Particle acceleration in solar flares is mostly explored by theoretical models, because neither macroscopic nor microscopic electric fields are directly measurable by remotesensing methods. The motion of particles can be described in terms of acceleration by parallel electric fields, drift velocities caused by perpendicular forces (i.e., $E \times B$-drifts), and gyromotion caused by the Lorentz force of the magnetic field. Theoretical models of particle acceleration in solar flares can be broken down into three groups: (1) DC electric field acceleration, (2) stochastic or second-order Fermi acceleration, and (3) shock acceleration (for an overview see Table 3; for references see Aschwanden 2004, p.470). In the models of the first group, there is a paradigm shift from large-scale DC electric fields (of the size of flare loops) to small-scale electric fields (of the size of magnetic islands produced by the tearing mode instability). The acceleration and trajectories of particles is studied more realistically in the inhomogeneous and time-varying electromagnetic fields around magnetic X-points and O-points of magnetic reconnection sites, rather than in static, homogeneous, large-scale Parker-type current sheets. The second group of models entails stochastic acceleration by gyroresonant wave-particle interactions, which can be driven by a variety of electrostatic and electromagnetic waves, supposed that wave turbulence is present at a sufficiently enhanced level and that the MHD turbulence cascading process is at work. The third group of acceleration models includes a rich variety of shock acceleration models, which is extensively explored in magnetospheric physics and could cross-fertilize solar flare models. Two major groups of models are studied in the context of solar flares (i.e., first-order Fermi acceleration or shock-drift acceleration, and diffusive shock acceleration).

New aspects are that shock acceleration is now applied to the outflow regions of coronal magnetic reconnection sites, where first-order Fermi acceleration at the standing fast shock is a leading candidate. Traditionally, evidence for shock acceleration in solar flares came mainly from radio type II bursts. New trends in this area are the distinction of different acceleration sites that produce type II emission: flare blast waves, the leading edge of CMEs (bowshock), and shocks in internal and lateral parts of CMEs. In summary 


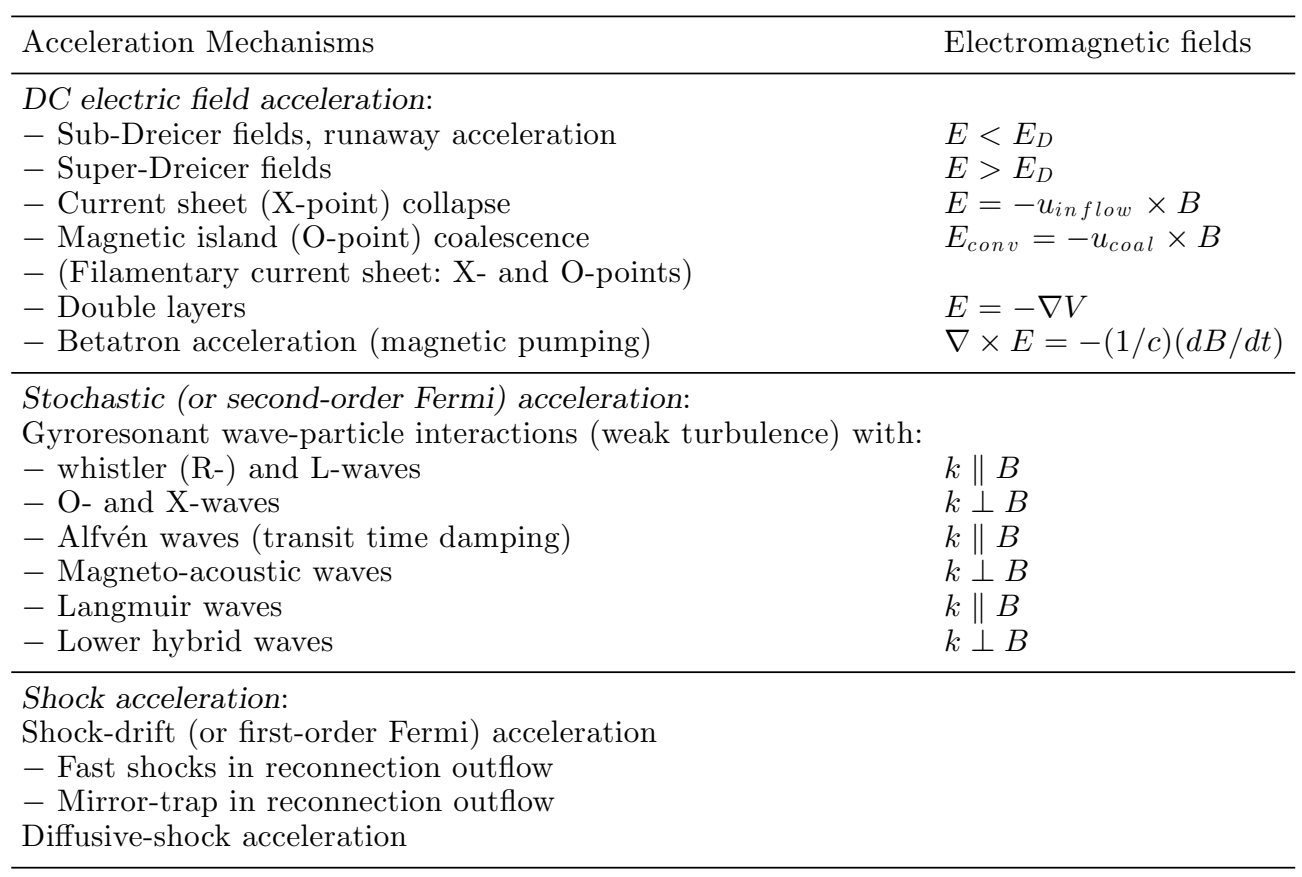

Table 3. Overview of particle acceleration mechanisms in solar flares (Aschwanden 2004).

we can say that (1) all three basic acceleration mechanisms seem to play a role to a variable degree in some parts of solar flares and CMEs, (2) the distinction between the three basic models become more blurred in more realistic (stochastic) models, and (3) the relative importance and efficiency of various acceleration models can only be assessed by including a realistic description of the electromagnetic fields, kinetic particle distributions, and MHD evolution of magnetic reconnection regions pertinent to solar flares.

Particle kinematics, the quantitative analysis of particle trajectories, has been systematically explored in solar flares by performing high-precision energy-dependent time delay measurements with the large-area detectors of the Compton Gamma-Ray Observatory (CGRO). There are essentially five different kinematic processes that play a role in the timing of nonthermal particles energized during flares: (1) acceleration, (2) injection, (3) free-streaming propagation, (4) magnetic trapping, and (5) precipitation and energy loss. The time structures of hard X-ray and radio emission from nonthermal particles indicate that the observed energy-dependent timing is dominated either by free-streaming propagation (obeying the expected electron time-of-flight dispersion) or by magnetic trapping in the weak-diffusion limit (where the trapping times are controlled by collisional pitch angle scattering). The measurements of the velocity dispersion from energy-dependent hard X-ray delays allows then to localize the acceleration region, which was invariably found in the cusp of postflare loops.

RHESSI observations produce new findings that challenge our previously established concepts. For instance, the acceleration and/or propagation of flare-accelerated electrons and ions seems to have distinctly different characteristics, since the $2.223 \mathrm{MeV}$ neutroncapture gamma-ray line was found to be significantly displaced from 200-200 keV hard $\mathrm{X}$ electrons (Hurford et al. 2003, 2006). Theoretical interpretations range from different acceleration path lengths for electrons and ions in the stochastic acceleration process 
(Emslie et al. 2004) to charge separation in the super-Dreicer electric field in a reconnecting non-neutral current sheet (Zharkova \& Gordovskyy 2004).

\section{Conclusions}

Our insights into the fundamental physical processes in the solar corona is exponentially growing thanks to all the new high-resolution imaging and spectral observations. We have identified almost all principal MHD wave modes in the solar corona, and theoretical studies are exploring now second-order effects, which provide previously unknown physical parameters. Turbulence is a process that is established in the lower solar atmosphere (driven by the subsurface magneto-convection), as well as in the solar wind (caused by interacting outgoing and reflected Alfvén waves), but seems to be less important in the closed-field corona (due to the large dissipation length of Alfvén waves). Magnetic reconnection is an ubiquitous process in the solar corona, ranging from small-scale phenomena in the transition region (explosive events, nanoflares, etc) to catastrophic large-scale reconfigurations during flares and CMEs, but modeling problems deal mostly with the relative importance of the drivers (magnetic flux emergence, cancellation, lossof-equilibrium, magnetic break-out, etc). The process of particle acceleration is, due to its microscopic physics, the most difficult to study, and most conclusions are drawn from secondary processes, such as the collisional thin- and thick-target interactions of the accelerated particles, or in-situ particle detections in interplanetary space. It is imperative to get a grip on the 3D geometry and topology of magnetic reconnection regions and shock waves in order to get a glimpse on the electromagnetic fields that accelerate highenergy particles. It is clear that only multi-wavelength observations with comprehensive modeling can lead to a deeper physical understanding and to more reliable conclusions about these fundamental physical processes in the solar corona. Conclusions about the operation of the same physical processes in stellar coronae can only be drawn by inference, but the sensitivity threshold of stellar observations generally imply a bias towards more energetic flare processes.

\section{Acknowledgements}

Part of this work was supported by NASA contracts NAG5-13490 (Living with a Star Targeted Research \& Technology), NNG06GC25G (Solar and Heliospheric Physics), NAS5-38099 (TRACE mission), and NAS5-98033 (RHESSI mission, through University of California, Berkeley, subcontract SA2241-26308PG).

\section{References}

Acton, L.W., Canfield, R.C., Gunkler, T.A., Hudson, H.S., Kiplinger, A.L., \& Leibacher,J.W. 1982, ApJ 263, 409

Aly, J.J. \& Amari,T. 1997, A\&A A, 319, 699

Andrews, A.D. 1990, A\&A 239, 235

Antiochos, S.K., DeVore, C.R., \& Klimchuk, J.A. 1999, ApJ 510, 485

Antonucci, E., Rosner, R., \& Tsinganos,K. 1986, ApJ 301, 975

Asai, A., Shimojo, M., Isobe, H., Morimoto, T., Yokoyama, T., Shibasaki, K., \& Nakajima, H. 2001, ApJ 562, L103

Asai, A., Yokoyama, T., Shimojo, M., \& Shibata,K. 2004, ApJ 605, L77

Asai, A., Yokoyama, T., Shimojo, M., Masuda, S., Kurokawa, H., \& Shibata, K. 2004a, ApJ 611,557

Aschwanden, M.J. 1987, Solar Phys. 111, 113

Aschwanden, M.J., Benz, A.O., \& Schwartz, R.A. 1993, ApJ 417, 790 
Aschwanden, M.J., Benz, A.O., Dennis, B.R., \& Schwartz, R.A. 1995, ApJ 455, 347

Aschwanden, M.J., Kosugi, T., Hudson, H.S., Wills, M.J., \& Schwartz,R.A. 1996, ApJ 470, 1198

Aschwanden, M.J., Fletcher, L., Schrijver, C., \& Alexander,D. 1999, ApJ 520, 880

Aschwanden, M.J., Kosugi, T., Hanaoka, Y., Nishio, M., \& Melrose,D.B. 1999a, ApJ 526, 1026

Aschwanden, M.J. 2001, ApJ 560, 1035

Aschwanden, M.J., Nakariakov, V.M., \& Melnikov,V.F. 2004, ApJ 600, 458

Aschwanden, M.J. 2004, Physics of the Solar Corona. New York: Springer

Aschwanden, M.J., Winebarger, A., Tsiklauri, D., \& Peter,H. 2007, ApJ 659, 1673

Berghmans, D. \& Clette, F. 1999, Solar Phys. 186, 207

Belcher, J.W. \& Davis, L. Jr. 1971, J. Geophys. Res. 76, 3534

Chae, J., Schuehle, U. \& Lemaire,P. 1998, ApJ 505, 957

Cranmer, S.R. \& VanBallegooijen, A.A. 2005, ApJS 156, 265

Czaykowska, A., DePontieu, B., Alexander, D., \& Rank,G. 1999, ApJ 521, L75

DeForest, C.E., \& Gurman, J.B. 1998, ApJ 501, L217

DeMoortel, I., Ireland, J., \& Walsh,R.W. 2000, A $8 A$ 355, L23

DeMoortel, I., Ireland, J., Walsh,R.W., \& Hood,A.W. 2002, Solar Phys. 209, 61 and 89

Dmitruk, P. \& Gomez, D.O. 1997, ApJ 484, L83

Dmitruk, P., Gomez, D.O., \& DeLuca,E.E. 1998, ApJ, 505, 974

Einaudi, G., Velli, M., Politano, H., \& Pouquet,A. 1996a, ApJ 457, L113

Einaudi, G., Califano, F., \& Chiuderi,C. 1996b, ApJ 472, 853

Emslie, A.G., Miller, J.A., \& Brown,J.C. 2004, ApJ 602, L69

Fletcher, L. \& Hudson,H.S. 2001, Solar Phys. 204, 71

Fletcher, L., Metcalf, T.R., Alexander, D., Ryder, L.A., \& Brown,D.S. 2001, ApJ 554, 451

Forbes, T.G. \& Acton,L.W. 1996, ApJ 459, 330

Forbes, T.G. \& Priest, E.R. 1995, ApJ 446, 377

Gary, D.E., Linsky, J.L., \& Dulk,G.A. 1982, ApJ 263, L79

Goldstein, M.L., Roberts, D.A., \& Matthaeus,W.H. 1995, Ann. Rev. Astron. Astrophys. 33, 283

Grigis, P.C. \& Benz, A.O. 2005, ApJ 625, L143

Hanaoka, Y. 1996, Solar Phys. 165, 275

Hanaoka, Y. 1997, Solar Phys. 173, 319

Heyvaerts,J. \& Priest,E.R. 1992, ApJ 390, 297

Heyvaerts, J., Priest, E.R., \& Rust,D.M. 1977, ApJ 216, 123

Hoyng, P., Duijveman, A., Machado, M.E., Rust, D.M., Svetska, Z., Boelee, A., DeJager, C., Frost, K.J., Lafleur, H., Simnett, G.M., VanBeek, H.F., \& Woodgate,B.E. 1981, ApJ 246, L155

Hurford, G.J., Schwartz, R.A., Krucker, S., Lin, R.P., Smith, D.M., \& Vilmer,N. 2003, ApJ 595, L77

Hurford, G.J., Krucker, S., Lin, R.P., Schwartz, R.A., Share, G.H., \& Smith,D.M. 2006, ApJ 644, L93

Inverarity, G.W., Priest, E.R., and Heyvaerts,J. 1995, A\&A 293, 913

Inverarity, G.W. \& Priest,E.R. 1995, A\&A 296, 395

Katsiyannis, A.C., Williams, D.R., McAteer, R.T.J., Gallagher, P.T., Keenan, F.P., \& Murtagh, F. 2003, A\&SA 406, 709

Kliem, B., Dammasch, I.E., Curdt, W., \& Wilhelm, K. 2002, ApJ 568, L61

Kopp, R.A. \& Pneuman, G.W. 1976, Solar Phys. 50, 85

Krucker, S., Hurford, G.J., \& Lin,u R.P. 2003, ApJ 595, L103

Krucker, S., Fivian, M.D., \& Lin, R.P. 2005, Adv. Space Res. 35, 1707

Leamon, R.J., Smith, C.W., Ness, N.,F., Matthaeus, W.H., \& Wong, H.K. 1998, JGR 103, 4775

Li, X., Habbal, S.R., Kohl, J.L., \& Noci,G. 1998, ApJ 501, L133

Mariska J.T. 2005, ApJ 620, L67

Mariska J.T. 2006, ApJ 639, 484

Masuda, S., Kosugi, T., Hara, H., Tsuneta, S., \& Ogawara,Y. 1994, Nature 371/6497, 495

Mathioudakis, M., Seiradakis, J.H., Williams, D.R., Avgoloupis, S., Bloomfield, D.S., and McAteer, R.T.J. 2003, A\&A 403, 1101

McKenzie, D.E. \& Hudson,H.S. 1999, ApJ 519, L93 
Melnikov, V.F., Shibasaki, K., \& Reznikova,V.E. 2002, ApJ 580, L185

Melnikov, V.F., Reznikova, V.E., Shibasaki, K., \& Nakariakov, V.M. 2005, Aש্AA 439, 727

Milano, L.J., Gomez, D.O., \& Martens,P.C.H. 1997, ApJ 490, 442

Milano, L.J., Dmitruk, P., Mandrini, C.H., Gomez, D.O., \& Demoulin, P. 1999, ApJ 521, 889

Mitra-Kraev, U., Harra, L.K., Williams, D.R., and Kraev, E. 2005, A\&3A 436, 1041

Mullan, D.J., Herr, R.B., Bhattacharyya,S. 1992 ApJ 391, 265

Mullan, D.J. \& Johnson, M. 1995, ApJ 444, 350

Nakariakov, V.M., Ofman, L., DeLuca, E., Roberts, B., \& Davila,J.M. 1999, Science 285, 862

Nakariakov, V.M., Melnikov, V.F., \& Reznikova, V.E. 2003, A\&A 412, L7

Nishio, M., Yaji, K., Kosugi, T., Nakajima, H., \& Sakurai,T. 1997, ApJ 489, 976

Robbrecht, E., Verwichte, E., Berghmans, D., Hochedez, J.F., Poedts,S., \& Nakariakov,V.M. 2001, A\& $A 370,591$

Rust, D.M., Simnett, G.M., \& Smith, D.F. 1985, ApJ 288, 401

Sakao, T., Kosugi, T., \& Masuda,S. 1998, "Observational Plasma Astrophysics: Five Years of Yohkoh and Beyond", (eds. Watanabe,T., Kosugi,T. \& Sterling,A.C.), Astrophysics and Space Science Library Vol. 229, Dordrecht: Kluwer, p.273

Sakao, T., Kosugi, T., Masuda, S., Yaji, K., Inda-Koide, M., \& Makishima, K. 1994, "New Look at the Sun with Emphasis on Advanced Observations of Coronal Dynamics and Flares", Nobeyama Radio Observatory Report No. 360, (eds. S.Enome and T.Hirayama), NOAJ, Japan, p.169

Sheeley, N.R., Wang, Y.M., Hawley, S.H., Brueckner, G.E., Dere, K.P., Howard, R.A. Koomen, M.J., Korendyke, C.M., Michels, D.J., Paswaters, S.E., Socker, D.G., StCyr, O.C., Wang, D., Lamy, P.L., Llebaria, A., Schwenn, R., Simnett, G.M., Plunkett, G.M., \& Biesecker,D.A. 1997, ApJ 484, 472

Shibata, K., Ishido, Y., Acton, L.W., Strong, K.T., Hirayama, T., Uchida, Y., McAllister, A.H., Matsumoto, R., Tsuneta, S., Shimizu, T., Hara, H., Sakurai, T., Ichimoto, K., Nishino, Y., \& Ogawara,Y. 1992, PASJ 44, L173

Sturrock, P.A. 1966, Nature, 5050, 695

Somov,B.V., Kosugi,T., \& Sakao,T. 1998, ApJ 497, 943

Sui, L. \& Holman, G.D. 2003, ApJ 596, L251

Svestka, Z., Fontenla, J.M., Machado, M.E., Martin, S.F., Neidig, D., \& Poletto,G. 1987, Solar Phys. 108, 237

Tomczyk, S., McIntosh, S.W., Keil, S.L., Judge, P.G., Schad, T., Seeley,D.H., \& Edmondson,J. 2007, Nature 317, 1192

Tsuneta, S., Hara, H., Shimizu, T., Acton, L.W., Strong, K.T., Hudson, H.S., \& Ogawara,Y. 1992, PASJ 44, L63

Tsuneta, S. 1996, ApJ 456, 840

Tsuneta, S., Masuda, S., Kosugi, T., \& Sato,J. 1997, ApJ 478, 787

Uchida,Y. 1980), "Solar Flares", A monograph from SKYLAB Solar Workshop II, (ed. P.A.Sturrock), Colorado Associated University Press, Boulder, Colorado, p.67 and 110

Verwichte, E., Nakariakov, V.M., \& Cooper,F.C. 2005, A\& A 430, L65

Wang, T.J., Solanki, S.K., Curdt, W., Innes, D.E., \& Dammasch,I.E. 2002, ApJ 574, L101

Williams, D.R., Phillips, K.J.H., Rudawy, P., Mathioudakis, M., Gallagher, P.T., O'Shea, E., Keenan, F.P., Read, P., Rompolt, B. 2001, MNRAS 326, 428

Wuelser, J.P., Canfield, R.C., Acton, L.W., Culhane, J.L., Phillips, A., Fludra, A., Sakao, T., Masuda, S., \& Kosugi, T. 1994, ApJ 424, 459

Yokoyama, T., Akita, K., Morimoto, T., Inoue, K., \& Newmark, J. 2001, ApJ 546, L69

Zarro, D.M. \& Canfield, R.C. 1989, ApJ 338, L33

Zharkova, V.V. \& Gordovskyy, M. 2004, ApJ 604, 884 